\title{
Coledocolitiasis como primera manifestación de agenesia vesicular
}

\section{Choledocholithiasis as the first manifestation of vesicular agenesis}

\author{
Juan José González de la Mora, ${ }^{*}$ Tania Karina Cázares Robles, ${ }^{*}$ Iván Alberto Nájera Rodríguez, * \\ Óscar Augusto Aguilar Soto, § Víctor Takeshi Landero Yoshioka*
}

\section{Resumen}

La ausencia congénita de vesícula biliar no es frecuente, tiene una incidencia de $0.02 \%$. La detección de agenesia vesicular suele ser incidental, se asocia a coledocolitiasis en $50 \%$ de los casos sintomáticos. Un hombre de 68 años con historia de ocho meses de ictericia y dolor abdominal intermitente reporta fiebre de una semana de duración. Se encuentra con ictericia generalizada, dolor abdominal en hipocondrio derecho y signo de Murphy negativo, además de elevación de la bilirrubina directa sérica. El ultrasonido y la colangiorresonancia magnética demostraron la dilatación de la vía biliar con un lito en el colédoco, sin visualizar la vesícula biliar. Tras realizarse una colangiopancreatografía retrógrada endoscópica (CPRE) sin éxito, se decidió un abordaje quirúrgico abierto con coledocotomía extrayendo un lito de $1.2 \mathrm{~cm}$. La colangiografía a través de un tubo en T demostró ausencia del conducto cístico y de la vesícula biliar. La agenesia vesicular puede representar un reto diagnóstico y terapéutico cuando se asocia con coledocolitiasis.

Palabras clave: Agenesia vesicular, coledocolitiasis, ictericia.

\section{INTRODUCCIÓN}

La ausencia congénita de vesícula biliar es una condición en extremo inusual, con una incidencia calculada entre 0.015 y $0.02 \% .^{1,2}$ Se ha descrito como conse-

* Cirujano General.

₹ Residente de Cirugía General, Universidad Autónoma Metropolitana Unidad Xochimilco.

$\S$ Cirujano General y Endoscopista.

Hospital Ángeles Pedregal. Ciudad de México.

Correspondencia:

Dr. Juan José González de la Mora

Correo electrónico: hap710@hotmail.com

Aceptado: 16-10-2019.

www.medigraphic.com/actamedica

\section{Abstract}

Congenital absence of gallbladder is unusual, with an incidence of $0.02 \%$. Gallbladder agenesis detection is mostly incidental, and when symptomatic, it is associated to choledocholithiasis in $50 \%$ of the cases. A 68 -year-old male, with a history of 8 months of intermittent jaundice and abdominal pain, reports fever for a week. Generalized jaundice, abdominal pain at right upper quadrant without Murphy's sign, and elevated serum direct bilirubin were evident. The ultrasound and the MRC showed bile duct dilatation, with a gallstone at de common bile duct, but no gallbladder. After unsuccessful ERCP, surgery with choledochotomy was decided to extract one $1.2 \mathrm{~cm}$ stone, and a T-tube cholangiogram showed the absence of cystic duct and gallbladder. Gallbladder agenesis, when associated with choledocholithiasis, can be a diagnostic and therapeutic challenge.

Keywords: Vesicular agenesis, choledocholithiasis, jaundice.

cuencia de alteraciones en el desarrollo embriológico alrededor de la quinta semana de gestación, por lo que puede estar asociada con otras malformaciones estructurales entre 40 y $70 \%$ de los casos. $^{2}$ Cuando no se acompaña de otras alteraciones, se clasifica en asintomática y al presentarse con ictericia, náusea, vómito y dolor en el cuadrante superior derecho, hasta en $90 \%$ de los casos, se denomina sintomática. ${ }^{1,3,4}$ Cuando la agenesia vesicular es asintomática, suele detectarse de manera incidental en autopsias, y más comúnmente durante la laparotomía exploradora. ${ }^{5,6}$ Debe considerarse como la causa menos probable para visualizar la vesícula biliar durante los estudios diagnósticos de imagen, ya que la presencia de colecistitis, errores técnicos y ciertas variantes anatómicas, así como fístulas cistocoledocianas (síndrome de Mirizzi) deben descartarse de primera instancia. ${ }^{2,6}$ Se ha reportado una 
asociación entre agenesia vesicular y coledocolitiasis en $50 \%$ de los casos sintomáticos, lo que confiere gran predisposición a colangitis ascendente, y en ocasiones representa un reto terapéutico. ${ }^{1,7}$

\section{CASO CLÍNICO}

Se presenta el caso de un hombre de 68 años, sin antecedentes quirúrgicos, diabético de larga evolución en control, con un cuadro de ocho meses caracterizado por ictericia y dolor tipo cólico en hipocondrio derecho

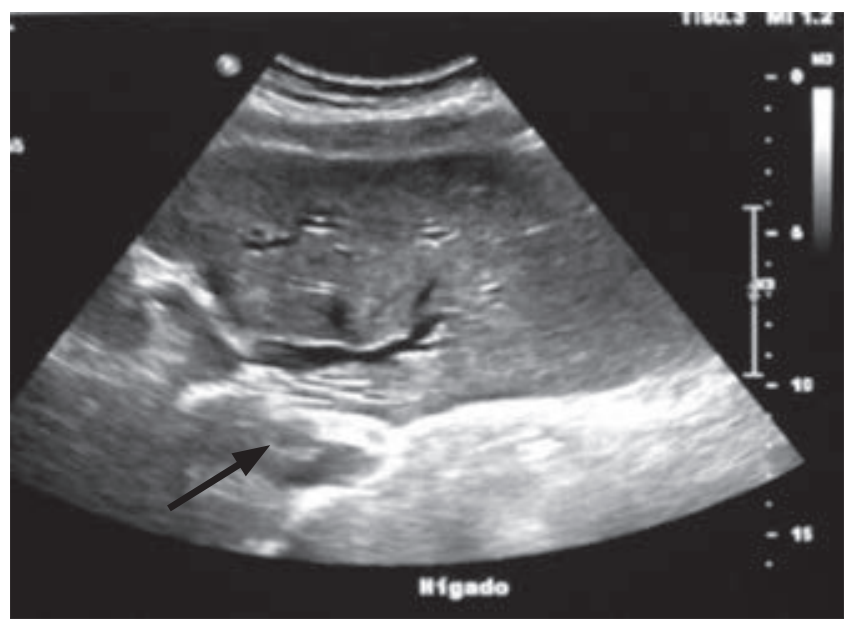

Figura 1: Ultrasonido de hígado y vías biliares. Vesícula biliar no visible, sin lesiones en parénquima hepático.

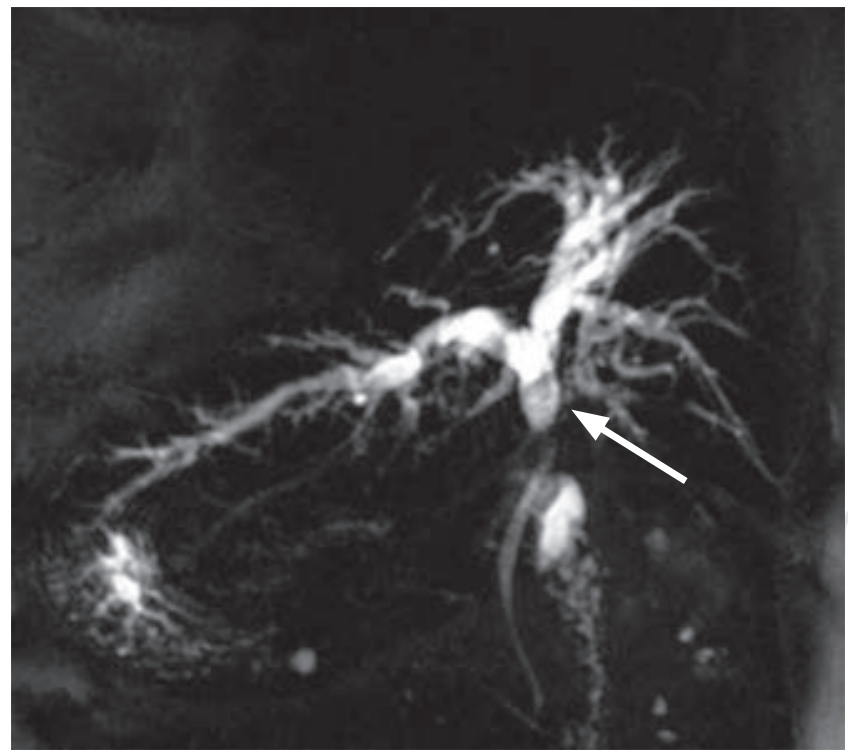

Figura 2: Colangiorresonancia magnética con dilatación de la vía biliar y ausencia de vesícula.

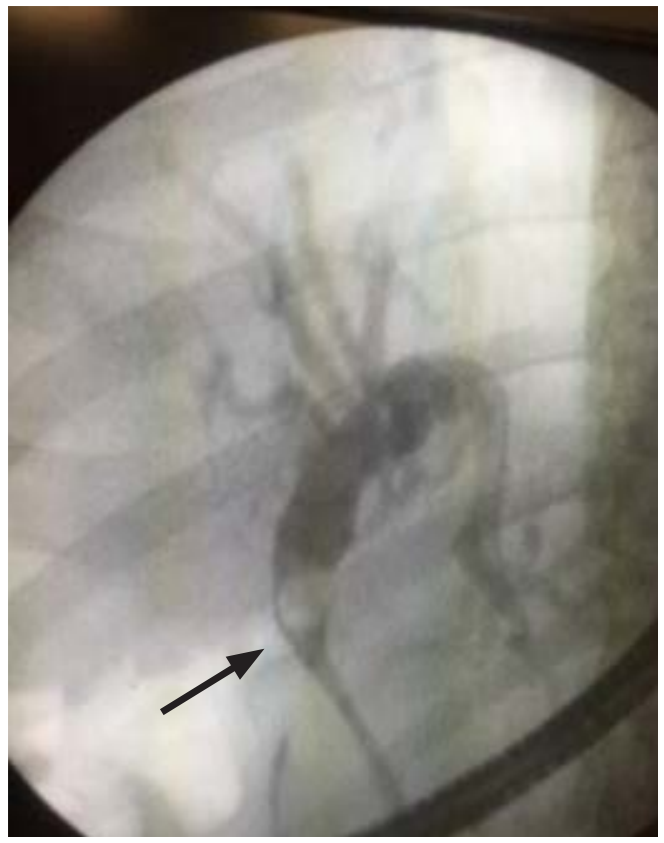

Figura 3: Colangiopancreatografía retrógrada endoscópica (CPRE). Lito a nivel suprapancrático y dilatación de vía biliar.

intermitente, de moderada intensidad, irradiado a la región subescapular, náusea, vómito, pérdida de peso, acolia y coluria. Se agregó fiebre, malestar general, astenia, adinamia y escalofrío en la última semana, por lo que acude a urgencias. Los signos vitales eran normales, con ictericia generalizada, deshidratación mucotegumentaria, abdomen doloroso a la palpación en hipocondrio derecho sin signo de Murphy ni hepatomegalia. Las pruebas de función hepática se mostraron alteradas asociadas con hiperbilirrubinemia a expensas de bilirrubina directa (bilirrubina total de $10.75 \mathrm{mg} /$ $\mathrm{dL}$ y bilirrubina directa de $8.04 \mathrm{mg} / \mathrm{dL}$ ). Tras abordaje médico y diagnóstico, el ultrasonido abdominal y la colangiorresonancia mostraron dilatación de la vía biliar y un colédoco de $1.5 \mathrm{~cm}$ de diámetro secundario a litos, respectivamente, ambos sin evidencia de vesícula biliar (Figuras 1 y 2).

La colangiopancreatografía retrógrada endoscópica (CPRE) fue fallida al no descomprimir la vía biliar a pesar de esfinterotomía (Figura 3).

Se decide abordaje quirúrgico con coledocotomía y extracción de diversos litos negros, el mayor con un diámetro de $1.2 \mathrm{~cm}$, se realizó colangiografía transoperatoria por sonda en T que demostró ausencia de conducto cístico y vesícula biliar (Figura 4). Se exploraron sitios alternativos de inserción de vesícula biliar, sin evidencia de ésta. 


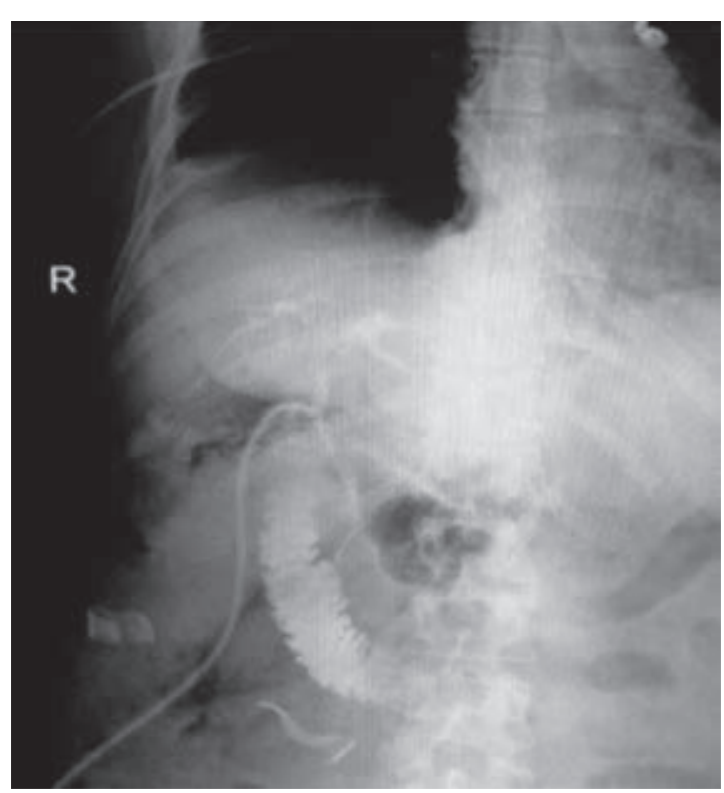

Figura 4: Colangiografía transoperatoria. Ausencia de conducto cístico y vesícula biliar.

\section{DISCUSIÓN}

La coledocolitiasis comúnmente se sospecha en escenarios de colelitiasis sintomática y en casos de pancreatitis aguda, con otras presentaciones como la producción de litos de novo en la vía biliar postcolecistectomía, que son menos frecuentes. Por lo anterior, la agenesia vesicular como causa de ictericia por coledocolitiasis representa un diagnóstico por descarte, retrasando el tratamiento definitivo y aumentando el riesgo de progresión a colangitis. De esta manera representa un reto en los casos refractarios, ya que propicia mayor morbilidad al realizar procedimientos diagnósticos y terapéuticos invasivos. ${ }^{1,8,9}$ Se han descrito diversos abordajes para su tratamiento, siendo la esfinterotomía, la dilatación papilar con balón y la extracción del lito con canasta por vía panendoscópica los abordajes más convencionales con tasas de éxito de 85 a 90\%. ${ }^{2}$ Otras medidas terapéuticas más avanzadas son la litotripsia electrohidráulica, litotripsia mecánica o con láser y la litotripsia por ondas de choque extracorpóreas; sin embargo, las complicaciones que surgen al realizar estos procedimientos son pancreatitis, ruptura y hemorragia duodenal, que al final requieren intervención quirúrgica en algunos casos. ${ }^{8}$ Anteriormente no existía ventaja alguna en realizar una CPRE preoperatoria en vez de una exploración de la vía biliar principal por cirugía abierta en ensayos controlados aleatorizados en sujetos de alto riesgo de coledocolitiasis. ${ }^{9}$ Sin embargo, actualmente la exploración abierta de vías biliares no se realiza con frecuencia dada la disminución de la morbilidad y el menor tiempo de estancia intrahospitalaria asociadas al abordaje por mínima invasión. ${ }^{9,10} \mathrm{En}$ este caso se optó por realizar una cirugía abierta por las dificultades previstas para la extracción del lito, previniendo así complicaciones propias del procedimiento como fuga por trayecto fistuloso y ruptura del colédoco que conllevan una morbilidad aproximada de $15 \%$ y mortalidad de hasta $6 \%$ reportadas en series grandes, además de la mejor exploración de la cavidad abdominal en busca de sitios de inserción de la vesícula biliar. ${ }^{10}$

\section{REFERENCIAS}

1. Tjaden J, Patel K, Aadam A. Gallbladder agenesis with refractory choledocholithiasis. Case Rep Gastrointest Med. 2015; 2015: 747931.

2. Trompetas V, Panagopoulos E, Ramantanis G. Gall-bladder agenesis presenting with obstructive jaundice and elevated CA 19-9. Acta Chir Bbelg. 2004; 104: 347-349.

3. Tang LM, Wang XF, Ren PT, Xu GG, Wang CS. The diagnosis of gallbladder agenesis: two cases report. Int J Clin Exp Med. 2015; 8 (2): 3010-3016.

4. Fiaschetti V, Calabrese G, Viarani S, Bazzochi G, Simonetti G. Gallbladder agenesis and cystic duct absence in an adult patient diagnosed by magnetic resonance cholangiography. Case Rep Med. 2009; 2009: 674768.

5. Sasaki K. Congenital agenesis of the gallbladder with the hypogenetic cystic duct definitely diagnosed by peroral cholangioscopy. Gastroenterol Pancreatol Liver Disord. 2014; 1 (3): 1-4.

6. Quintana AB, Díaz FJ, Lamikiz AV, Valdevieso AL, Perdigo L. Ausenciadesaparición de la vesícula biliar en un paciente diagnosticado de colelitiasis. Cir Esp. 2005; 78 (5): 331-332.

7. Waisberg J, Pinto PE, Gusson PR, Fasano PR, de Godoy AC. Agenesis of the gallbladder and cystic duct. Sao Paulo Med J. 2002; 120 (6): 192-194.

8. Toshihide O, Susumo T. Complication of endoscopic therapeutic lithotripsy for choledocholithiasis. JJBA. 2010; 24: 754-761.

9. Maple JT, Ben-Menachem T, Anderson MA, Appalaneni V, Banerjee S, Cash BD et al. The role of endoscopy in the evaluation of suspected choledocholithiasis. Gastrointest Endosc. 2010; 71 (1): 1-9.

10. Garteiz-Martínez D, Weber-Sánchez A, López-Acosta ME. Laparoscopic T-tube choledochotomy for biliary lithiasis. JSLS. 2008; 12: 326-331. 